\title{
Chloroquine treatment against naturally occuring Giardia duodenalis infection in dogs ${ }^{*}$
}

\author{
KEREM URAL, MEHMET GULTEKIN, SERDAR PASA, NURAN AYSUL*, ADNAN AYAN*
}

Department of Internal Medicine, *Department of Parasitology, Faculty of Veterinary, Adnan Menderes University, Isikli, Aydin, Turkey

The aim of the present study was to evaluate the efficacy of chloroquine on lessening or eliminating cyst shedding in dogs naturally infected with Giardia duodenalis. A total of 26 dogs naturally infected with G. duodenalis, from various breeds, ages and of both sexes were selected and randomly assigned into two groups based on treatment (group $\mathrm{I}, \mathrm{n}=\mathbf{2 0}$ dogs treated orally with $2.5 \mathrm{mg} / \mathrm{kg}$ chloroquine twice daily for 5 consecutive days) and control (group II, $n=6$ untreated control dogs). Diagnosis was based on microscopic faecal examination and rapid test kits. Cyst excretion was determined on days $0,3,7$ and 10, before and after treatment. Evaluation of the percentage reduction in cyst excretion revealed $99.8 \%$ efficacy on day 3 and $99.9 \%$ on days 7 and 10 in the treatment group. Geometric means of the number of excreted cysts did not change significantly in the control group. Chloroquine at the proposed dosage might be a practical in application, low priced and highly effective treatment option in dogs with giardiasis.

Keywords: Giardia duodenalis, dog, chloroquine, treatment

Giardia duodenalis (syn. G. intestinalis, G. lamblia), an endemic and enteric flagellated protozoan parasite, has commonly been encountered in humans and animals worldwide (30). The prevalence rates for Giardia infection in dogs and cats may change depending on the population, area, diagnostic method, and health status of the animal and is commonly distibuted from $5 \%$ to $15 \%$ (31). Higher levels of infection, up to $100 \%$, have been reported in younger dogs housed in kennels, breeding operations, and research facilities (2).

Giardia infections in dogs are not usually accompanied by clinical signs, although giardiasis may cause anorexia, weight loss, lethargy, vomiting and enteritis with acute or chronic diarrhea (31). Diarrhea is mainly caused by intestinal malabsorption and hypersecretion (8). Younger or immunosuppressed animals have been reported to be more susceptible to disease (12).

The role of dogs as a source of human giardiasis seems to be unclear: several reports are divergent about the zoonotic potential. Epidemiological and molecular studies revealed that $G$. duodenalis is composed by at least seven assemblages (A to G). Genotypes of assemblages $\mathrm{A}$ and $\mathrm{B}$ are mainly known to infect humans (2,

*) This study was summarized partially from a national Project and was funded by Adnan Menderes University Research Projects Funding Unit with project number VTF-13009. The researchers who put pen and great effort to this study were involved.
18). Assemblages $C$ and $D$ were determined as speciesspecific to dogs (30). However, DNA of assemblages $\mathrm{A}$ and $\mathrm{B}$ were also described in dogs (25).

The high prevalence rates, undesirable clinical findings, and potential zoonotic risk warrant suitable treatment of giardiasis in dogs. Although there is no approved veterinary drug on the market, several compounds such as drugs in the nitroimidazole $(1,16)$ and benzimidazole class $(4,5)$, febantel-praziquantel-pyrantel combinations (3), azithromycin (35), silymarin (10) have reported effectiveness in reducing the level of cyst shedding and clinical signs. Metronidazole seems to be the first line drug for the treatment of giardiasis by most of veterinary clinicians (31). Albeit it has been concluded that controlled studies are lacking and administration of metronidazole has been associated with central nervous system toxicity in dogs $(11,17)$. Moreover, inefficacy of metronidazole is commonly reported in the treatment of giardiasis, and reinfection has been compromised as the most common cause of treatment failure (29). Moreover, there is an increasing number of treatment failures reported in human medicine (14). Thus, both veterinary and human medicine are seeking new therapeutic options.

The aim of the present study was to determine the efficacy of chloroquine, a 4-aminoquinoline compound 
synthetic agent that has been recognized as an old drug with a new perspective against giardiasis (14), to those dogs naturally infected with $G$. duodenalis.

\section{Material and methods}

Animal population. The present study was performed among 26 dogs reffered to the Adnan Menderes University, Faculty of Veterinary, Department of Internal Medicine located in Aydin city, Eagean Region of Turkey. Animals from different breeds, aged 3 to 11 months, and of both sexes (14 female and 12 male) were selected to those of presenting clinical signs compatible with a suspectible Giardia infection such as diarrhea, abdominal pain and/or vomitting.

Prior to the study design, a total of 95 dogs suspected of Giardia infection were analyzed with rapid test kits (SNAP Giardia test, IDEXX Veterinary Diagnostics, United States). Out of those 95 analyzed cases, dogs with naturally occuring giardiasis $(\mathrm{n}=26)$ were randomly assigned into two groups. Group I $(\mathrm{n}=20)$ involved chloroquine (Kutlu $250 \mathrm{mg}$ tablet $^{\mathbb{R}}$, Keymen Ilac San. Tic. Ltd. Sti, Turkey) treatment orally at a dose of $2.5 \mathrm{mg} / \mathrm{kg}$ twice daily for 5 consecutive days; Group II $(n=6)$ were left as controls and received placebo. Chloroquine was applied directly into the mouth, followed by $5 \mathrm{ml}$ water by the investigator; the placebo included an equivalent volume of water. General health control was carried out and faecal samples were collected on days 3, 7 and 10 after the first administration.

Both groups of dogs were consuming commercially prepared dog food and were housed in individual pens having separate facilities for preventing cross-contamination during the study. The pens where the cases were kept were cleaned and disinfected with a product containing quaternary ammonium (Derdevice Plus Y, Deren Ilac, Turkey) for elimination of existing environmental parasitic contamination.

The study protocol was approved by the institutional laboratory animals ethics committee of Adnan Menderes University HADYEK (with no: 2012/088 and date 04.10.2012). Prior to enrolment in the present study, informed written consent was obtained from all of the owners/animal care takers. Taking into account ethical concerns, only a limited number of dogs served as controls. Albeit at the end of the trial, all positive control dogs were also treated with chloroquine at the same dosage to the previously treated animals.

Laboratory analysis. After initial physical examination, all dogs were screened on days $0,3,7$, and 10 to confirm the presence/absence of $G$. duodenalis cysts (and for other possible intestinal parasites relevant to dogs, i.e. Cryptosporidium $s p$. oocysts in the faeces). On days 0 and 10 hematological (WBC, RBC, HCT, MCHC, PLT) and serum biochemical (ALT, AST, creatinine, triglyceride, urea) values were determined.

Faecal examination. Faecal samples were collected manually from the rectum of all dogs on days 0 (before treatment), 3, 7 and 10 (after treatment). Furthermore $1.5 \mathrm{~g}$ of faecal material was mixed with $33 \% \mathrm{ZnSO}_{4}$ solution $(15 \mathrm{ml})$ and strained onto centrifuge tubes, which then was centrifuged at $880 \times g$ for 5 minutes, similary to what has been described elsewhere (34). The latter procedure was followed by collection of a $50 \mu \mathrm{l}$ of the supernatant, which was then placed on a microscope slide with Lugol iodine, covered by a slip. The slide was examined microscopically under $400 \times$ power for detection of Giardia cysts. Afterwards this step was repeated twice from different samples belonging to each dog by a single blind researcher. The number of cysts per gram of faeces $(\mathrm{CPG})$ was calculated by $[($ number of cysts identified $\times 100) / 1.5]$.

Assessment of treatment efficacy. Chloroquine treatment efficacy in the present study was assessed by microscopic examination of faecal samples collected on days 0,3 , 7 and 10, and percentage of reduction in CPG for the treatment group compared to the control group. The percentage of reduction in cyst excretion was calculated by use of the Henderson-Tilton formula (23), including geometric mean of CPG similar to those of Geurden et al. (19) which was also used by Ural et al. (34): $100 \times[1-(\mathrm{Ta} \times \mathrm{Cb}) /(\mathrm{Tb} \times \mathrm{Ca})]$.

$\mathrm{Ta}$ and $\mathrm{Tb}$ represented the geometric mean of $\mathrm{CPG}$ in the chloroquine treatment group before $(\mathrm{Tb})$ and after $(\mathrm{Ta})$ treatment, whereas $\mathrm{Ca}$ and $\mathrm{Cb}$ represented before $(\mathrm{Cb})$ and after $(\mathrm{Ca})$ placebo administration in the control animals.

Statistical analysis. Statistical analyses were performed using the SPSS statistical software package (version 22; SPSS Inc., Chicago, IL). The results for CPG in both control and treatment groups were tested for normality using the Kolmogorov-Smirnov test. The CPG was not normally distributed. Related samples: Friedman's two way-analysis of variance test was done before (day 0 ) and after the start of treatment (days 3, 7, 10) for each group. A Mann-Whitney$\mathrm{U}$ test was used to compare differences between groups for each day. Probability $(P)$ values: $<0.05$ was considered to indicate a significant difference.

\section{Results and discussion}

Cyst excretion. The results of the cyst counts were presented in Table 1. Throughout the study period dogs in the control group remained positive; moreover 3 out of 6 dogs presented an increase in cyst counts on day 10 (ranged between 150 000-300 600 CPG) compared to the initial values (100 000-300 $300 \mathrm{CPG}$ ), albeit there was no statistical significance. Treatment with chloroquine reduced cyst shedding in all cases on day 3 $(99.98 \%)$, increase in the rate of reduction of cyst shedding (99.99\%) and the absence of cyst excretion were detected in 11 patients on day 7 , reduction of the cyst shedding was continuing (99.99\%) and the absence of cyst excretion were determined in 16 cases on day 10 .

Tab. 1. The geometric means of the number of the excreted CPG in the control and chloroquine treated groups at each sampling day (before treatment [day 0] and after treatment [days $3,7,10])$. The percentage of reduction calculated based on geometric means is presented.

\begin{tabular}{|l|c|c|c|c|}
\hline & Day 0 & Day 3 & Day 7 & Day 10 \\
\hline Control $(\mathrm{n}=6)$ & $207918^{\mathrm{a}}$ & $142893^{\mathrm{a}}$ & $52287^{\mathrm{a}}$ & $223765^{\mathrm{a}}$ \\
Treatment $(\mathrm{n}=20)$ & $165293^{\mathrm{a}}$ & $315^{\mathrm{b}}$ & $125^{\mathrm{b}}$ & $7,7^{\mathrm{b}}$ \\
$\mathrm{p}$ value & 0.381 & 0.0001 & 0.002 & 0.0001 \\
$\begin{array}{l}\text { Reduction in cyst } \\
\text { excretion }\end{array}$ & - & $99.98 \%$ & $99.99 \%$ & $99.99 \%$ \\
\hline
\end{tabular}

Explanations: $\mathrm{a}, \mathrm{b}$-different letters indicate significant differences between rows and columns $(p<0.05)$. 
Distribution of cyst number in the treatment group before treatment ranged from 105 000-310 000 CPG; cyst number reduced gradually following chloroquine treatment on days 3 (0-20 000 CPG), 7 (0-15 000 CPG) and 10 (0-7200 CPG).

Hematological and serum biochemical analysis. There was no statistically significant difference in hematological and serum biochemical variables on days 0 and 10 between the groups studied (data was not shown).

Treatment applications. Dogs in both groups had clinical signs compatible with naturally occuring giardiasis, involving diarrhea on day 0 . No observable and significant side effects to chloroquine application were detected in the treatment group during the study. Neither coccidosis nor cryptosporidiosis infection were found in any of the animals enrolled throughout the study period.

G. duodenalis has been noticed as a significant and common intestinal pathogen. Relatively few choices are commercially available for anti-giardial treatment and there is no approved veterinary drug on the market (31). 5-nitroimidazole compounds such as metronidazole are first line anti-giardial treatment choice in human medicine (9). However, cases refractory to treatment applications within the latter compounds are becoming frequent (14). Several drugs have been tested against giardia infections in dogs $(3-5,10,16$, 35 ) but metronidazole is used routinely for treatment of giardiasis $(16,31)$. However, controlled studies are lacking in veterinary medicine and administration of metronidazole has been associated with toxicities $(11,17)$. Therefore, it is prudent to suggest that there is a need for searching for new treatment choices both in human beings and in the veterinary field.

Chloroquine, an old but promising agent, has now been recognized as an old drug with a new perspective against giardiasis (13). Chloroquine, a 4-aminoquinoline compound, is a synthetic agent. The latter compund was first synthesized at the Bayer laboratories in 1934, modified from quinacrine via replacing its acridine ring by a quinoline ring (13). For a good number of years, chloroquine was used as a first line option for the vast majority of treatments of malaria. Currently it has widely been used in many locations of the world as a reliable treatment against uncomplicated malaria (13). Furthermore the usage of this drug has not been limited to malaria, since it is recommended as a second-line treatment option for other infections, such as HIV (7), sarcoidosis (15), amoebiasis (28) and noninfectious diseases in humans (14).

In a prior study, in vitro activity of chloroquine against 25 isolates of $G$. duodenalis trophozoites demonstrated that more than half of the isolates were extremely susceptible (21). Furthermore, chloroquine was shown to be more effective than metronidazole on G. duodenalis trophozoites (26). Although the mechanism of action of chloroquine is not entirely under- stood, as the latter compound's efficacy was attributed to a reduced ability of Giardia trophozoite in vitro for attaching to surfaces. Hence it was also claimed that in vivo conditions possessed the existence of a similar effect (6). Chloroquine has also been recognized as a lysosomotropic agent. Lysosomes prevent endosomal acidification that cause the inhibition of endocytosis, degradation, recycling, and secretion of protein (32). Giardia exhibits peripheral vacuoles rather than a defined endosomal/lysosomal system. It may be suggested that the latter compound might inhibit peripheral vacuoler functions (33). These mechanisms might be related to the high efficacy against $G$. duodenalis in the present study.

The efficacy of chloroquine in dogs is comparable to what has been described in lambs and calves naturally infected with giardiasis, in which an oral dose of $2.5 \mathrm{mg} / \mathrm{kg}$ during 5 consecutive days resulted in $100 \%$ efficacy, as no cysts were found in faeces after treatment $(22,24)$. In the present study chloroquine treatment significantly reduced the cyst excretion by $99.8 \%$ on day 3 , afterwards $99.9 \%$ on days 7 and 10 .

Cleaning and disinfection of the environment are recommended to reduce the re-infection risk $(19,20)$. Studies showed that dogs re-excreted cysts shortly after the end of treatment (20). In addition, giardia cysts may survive more than a month in soil (27). Therefore, treatment protocols should be combined with disinfection of the environment (20). In our study, individual boxes of dogs were cleaned and disinfected every day with a quaternary ammonium product. No re-infection was determined on days 7 and 10 in the treatment group. However, it may be suggested that a long term followup is necessary to evaluate re-infection.

The high cyst reducing activity of chloroquine against giardiasis may provide an important benefit in private veterinary clinics or other veterinary service facilities (34). The easily availability of this cheap drug on the market, its cost (approximately 0.05 dollars per dog for 5 days treatment), have been significant impediments for usage of this antimalarial drug against giardiasis in dogs.

In conclusion chloroquine might be a practically appliable, reasonably priced and highly effective treatment option in dogs with giardiasis. Moreover, side effects in dogs receiving higher doses should be evaluated both in healthy and diseased subjects. Further studies with different doses and durations of treatment in a larger and longer study might be beneficial.

\section{References}

1. Abbitt B., Huey R. L., Eugster A. K., Syler J.: Treatment of giardiasis in adult greyhounds, using ipronidazole-medicated water. J. Am. Vet. Med. Assoc. 1986, 188, 67-69.

2. Ballweber L. R., Xiao L., Bowman D. D., Kahn G., Cama V. A.: Giardiasis in dogs and cats: update on epidemiology and public health significance. Trends Parasitol. 2010, 26, 180-189.

3. Barr S. C., Bowman D. D., Frongillo M. F., Joseph S. L.: Efficacy of a drug combination of praziquantel, pyrantel pamoate, and febantel against giardiasis in dogs. Am. J. Vet. Res. 1998, 59, 1134-1136. 
4. Barr S. C., Bowman D. D., Heller R. L.: Efficacy of fenbendazole against giardiasis in dogs. Am. J. Vet. Res. 1994, 55, 988-990.

5.Barr S. C., Bowman D. D., Heller R. L., Erb H. N.: Efficacy of albendazole against giardiasis in dogs. Am. J. Vet. Res. 1993, 54, 926-928.

6. Baveja U. K., Bathia V. N., Warhurst D. C.: Giardia lamblia: in-vitro sensitivity to some chemotherapeutic agents. J. Commun. Dis. 1998, 130, 79-84.

7. Brouwers J., Vermeire K., Schols D., Augustijns P.: Development and in vitro evaluation of chloroquine gels as microbicides against HIV-1 infection. Virology 2008, 378, 306-310.

8. Buret A. G.: Pathophysiology of enteric infections with Giardia duodenalis. Parasite 2008, 15, 261-265.

9. Busatti H. G. N. O., Santos J. F. G., Gomes M. A.: The old and new therapeutic approaches to the treatment of giardiasis: Where are we? Biologics 2009, 3 , 273-287.

10. Chon S. K., Kim N. S.: Evaluation of silymarin in the treatment on asymptomatic Giardia infections in dogs. Parasitol. Res. 2005, 97, 445-451.

11. Dow S. W., Le Couteur R. A., Poss M. L., Beadleston D.: Central nervous system toxicosis associated with metronidazole treatment of dogs: five cases (1984-1987). J. Am. Vet. Med. Assoc. 1989, 195, 365-368.

12. Epe C., Coati N., Schieder T.: Results of parasitological examinations of fecal samples from horses, ruminants, pigs, dogs, cats, hedgehogs and rabbits between 1998 and 2002. Dtsch. Tierarztl. Wochenschr. 2004, 111, 243-247.

13. Escobedo A. A., Almirall P., Cimerman S., Lalle M., Pacheco F., Acanda C. Z., Sánchez N.: Chloroquine: an old drug with new perspective against Giardiasis. Recent Pat. Antiinfect. Drug Discov. 2015, 10, 134-141.

14. Escobedo A. A., Ballesteros J., González-Fraile E., Almirall P.: A meta-analysis of the efficacy of albendazole compared with tinidazole as treatments for Giardia infections in children. Acta Trop. 2016, 153, 120-127.

15. Fazzi P.: Pharmacotherapeutic management of pulmonary sarcoidosis. Am. J. Respir. Crit. Care Med. 2003, 2, 311-320.

16. Fiechter R., Deplazes P., Schnyder M.: Control of Giardia infections with ronidazole and intensive hygiene management in a dog kennel. Vet. Parasitol. 2012, 187, 93-98.

17. Fitch R., Moore M., Roen D.: Metronidazole neurotoxicity in a dog. Prog. Vet Neurol. 1992, 307-309.

18. Geurden T., Olson M. E.: Giardia in pets and farm animals, and their zoonotic potential, [in:] Lujàn H. D., Svärd S. (ed.): Giardia - A Model Organism. Springer, Wien, New York 2011, p. 71.

19. Geurden T., Pohleb H., Sarrea C., Dreesena L., Vercruyssea J., Claerebouta E. The efficacy of a treatment with fenbendazole against an experimental Giardia duodenalis infection in lambs. Small Rumin. Res. 2011, 96, 211-215.

20. Geurden T., Vercruysse J., Claerebout E.: Field testing of a fenbendazole treatment combined with hygienic and management measures against a natural Giardia infection in calves. Vet. Parasitol. 2006, 142, 367-371.
21. Gordts B., Hemelhof W., Asselman C., Butzler J.: In vitro susceptibilities of 25 Giardia lamblia isolates of human origin to six commonly used anti-protozoal agents. Antimicrob. Agents Chemother. 1985, 28, 378-380.

22. Gultekin M., Ural K., Aysul N., Ayan A., Balikci C., Akyildiz G.: The efficacy of chloroquine treatment against naturally occurring Giardia duodenalis infection in calves. Vlaams Diergeneeskd. Tijdschr. 2016, 85, 335-341.

23. Henderson C. F., Tilton E. W.: Tests with acaricides against the brown wheat mite. J. Econ. Entomol. 1955, 48, 157-161.

24. Karademir U., Ural K., Aysul N., Ayan A., Toplu S., Ortlek O., Balikci C., Künyeli A., Erdoğan H.: Chloroquine treatment against Giardiasis in lambs. Rev. MVZ Cordoba 2016, 21, 5328-5335.

25. Lalle M., Jimenez-Cardosa E., Caccio S. M., Pozio E.: Genotyping of Giardia duodenalis from humans and dogs from Mexico using a $\beta$-giardin nested polymerase chain reaction assay. J. Parasitol. 2005, 91, 203-205.

26. Nava-Zuazo C., Estrada-Soto S., Guerrero-Álvarez J., León-Rivera I. Molina-Salinas G. M., Said-Fernández S., Navarrete-Vazquez G.: Design, synthesis, and in vitro antiprotozoal, antimycobacterial activities of $\mathrm{N}-\{2-$ [(7-chloroquinolin-4-yl) amino] ethyl\} ureas. Bioorg. Med. Chem. 2010, 18, 6398-6403.

27. Olson M. E., Goh J., Phillips M., Guselle N., McAllister T. A.: Giardia cyst and Cryptosporidium oocyst survival in water, soil, and cattle feces. J. Environ. Qual. 1999, 28, 1991-1996.

28. Reed S. L.: Amebiasis: an update. Clin. Infect. Dis. 1992, 14, 385-393.

29. Saffar M. J., Qaffari J., Khalilian A. R., Kosarian M.: Rapid reinfection by Giardia lamblia after treatment in a hyperendemic area: the case against treatment. East. Mediterr. Health J. 2005, 11, 73-78.

30. Scorza A. V., Ballweber L. R., Tangtrongsup S., Panuska C., Lappin M. R. Comparisons of mammalian Giardia duodenalis assemblages based on the $\beta$-giardin, glutamate dehydrogenase and triose phosphate isomerase genes. Vet. Parasitol. 2012, 189, 182-188

31. Scorza V., Lappin M. R.: Giardiasis, [in:] Greene C. E. (ed.): Infectious Diseases of the Dog and Cat. Elsevier/Saunders, St. Louis 2012, p. 785.

32. Steinman R. M., Mellman I. S., Muller W. A., Cohn Z. A.: Endocytosis and the recycling of plasma membrane. J. Cell Biol. 1983, 96, 1-27.

33. Tai J. H., Ong S. J., Chang S. C., Su H. M.: Giardiavirus enters Giardia lamblia WB trophozoite via endocytosis. Exp. Parasitol. 1993, 76, 165-174.

34. Ural K., Aysul N., Voyvoda H., Ulutas B., Aldemir O. S., Eren H.: Single dose of secnidazole treatment against naturally occuring Giardia duodenalis infection in Sakiz lambs. Rev. MVZ Cordoba 2014, 19, 4023-4032.

35.Zygner W., Jaros D., Gójska-Zygner O., Wedrychowicz H.: Azithromycin in the treatment of a dog infected with Giardia intestinalis. Pol. J. Vet. Sci. 2007, $11,231-234$.

Corresponding author: Mehmet Gultekin, DVM, PhD; Department of Internal Medicine, Faculty of Veterinary, Adnan Menderes University, Isikli, Aydın, 09017, Turkey; e-mail: gultekinmehmet@gmail.com 